\title{
The Genetic Basis of Hypertriglyceridemia
}

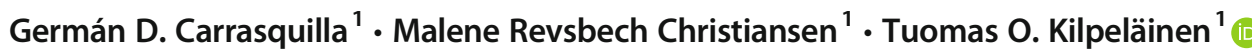

Accepted: 17 May 2021 / Published online: 19 June 2021

(C) The Author(s) 2021

\begin{abstract}
Purpose of Review Hypertriglyceridemia is a common dyslipidemia associated with an increased risk of cardiovascular disease and pancreatitis. Severe hypertriglyceridemia may sometimes be a monogenic condition. However, in the vast majority of patients, hypertriglyceridemia is due to the cumulative effect of multiple genetic risk variants along with lifestyle factors, medications, and disease conditions that elevate triglyceride levels. In this review, we will summarize recent progress in the understanding of the genetic basis of hypertriglyceridemia.

Recent Findings More than 300 genetic loci have been identified for association with triglyceride levels in large genome-wide association studies. Studies combining the loci into polygenic scores have demonstrated that some hypertriglyceridemia phenotypes previously attributed to monogenic inheritance have a polygenic basis. The new genetic discoveries have opened avenues for the development of more effective triglyceride-lowering treatments and raised interest towards genetic screening and tailored treatments against hypertriglyceridemia.

Summary The discovery of multiple genetic loci associated with elevated triglyceride levels has led to improved understanding of the genetic basis of hypertriglyceridemia and opened new translational opportunities.
\end{abstract}

Keywords Hypertriglyceridemia $\cdot$ Triglycerides $\cdot$ Dyslipidemia $\cdot$ Human genetics $\cdot$ Genetic variant $\cdot$ Monogenic $\cdot$ Polygenic

\section{Introduction}

Hypertriglyceridemia, defined as plasma triglyceride levels $>1.7$ $\mathrm{mmol} / \mathrm{L}(150 \mathrm{mg} / \mathrm{dL})$, affects about $10 \%$ of the adult population and it is thus the most common form of dyslipidemia worldwide $[1,2]$. Patients with hypertriglyceridemia are at an increased risk of stroke and coronary heart disease [3-6] and, in severe cases, may develop acute pancreatitis [7-9]. It is still debated whether

Germán D. Carrasquilla and Malene Revsbech Christiansen contributed equally to this work.

This article is part of the Topical Collection on Genetics and Genomics

Tuomas O. Kilpeläinen

tuomas.kilpelainen@sund.ku.dk

Germán D. Carrasquilla

german.carrasquilla@sund.ku.dk

Malene Revsbech Christiansen

malene.christiansen@ sund.ku.dk

1 Novo Nordisk Foundation Center for Basic Metabolic Research, Faculty of Health and Medical Sciences, University of Copenhagen, Mærsk Building, Blegdamsvej 3B, 2200 Copenhagen, Denmark triglycerides directly promote cardiovascular disease or represent a biomarker for the increased levels of atherogenic, triglyceriderich lipoproteins [10, 11]. Hypertriglyceridemia is often concomitant with other conditions, such as obesity, type 2 diabetes, and the metabolic syndrome, which contribute to the associated morbidity, mortality, and health care costs [9].

The clinical diagnosis of hypertriglyceridemia is based on fasting triglyceride levels, but non-fasting plasma triglyceride levels can also be applied [8,11-13]. There is no global consensus on the threshold values for classification of hypertriglyceridemia. For the purposes of the present review, we will apply the guidelines of the European Atherosclerosis Society and the European Society of Cardiology that categorize hypertriglyceridemia into mild-to-moderate (1.7-10 $\mathrm{mmol} / \mathrm{L})$ and severe (> $10 \mathrm{mmol} / \mathrm{L})$ types [11]. Mild-tomoderate hypertriglyceridemia indicates a triglyceride level at which cardiovascular disease risk is increased, and severe hypertriglyceridemia indicates an increased risk of acute pancreatitis [11]. Severe hypertriglyceridemia is uncommon, observed in $\sim 0.01 \%$ of the general population [14] and $\sim 1-2 \%$ of all hypertriglyceridemic adults $[1,2]$.

Genetic mechanisms that predispose to hypertriglyceridemia affect the synthesis, metabolism, or clearance of 
triglycerides, leading to increased levels of triglycerides in the blood [15] (Fig. 1, Table 1). Severe hypertriglyceridemia that appears at a young age is often due to monogenic causes [16•]. However, the vast majority of hypertriglyceridemias in the adult population have a complex polygenic basis where the cumulative effect of multiple independent variants, together with non-genetic factors, elevates triglyceride levels [10] (Fig. 2). During the last decade, genome-wide association studies (GWASs) have uncovered more than 300 independent genetic loci [17-22, 23••, 24-33] associated with plasma triglyceride levels, opening new avenues for early prediction of hypertriglyceridemia and the development of more effective treatments [34].

In this review, we will outline recent progress in the understanding of the genetic underpinnings of hypertriglyceridemia.

\section{Monogenic Hypertriglyceridemia}

\section{Familial Chylomicronemia Syndrome}

In familial chylomicronemia syndrome (FCS), an autosomal recessive or compound heterozygous variant in a critical gene regulating chylomicron catabolism is found $[1,8,16 \bullet]$, leading to high chylomicron levels and low levels of other lipoprotein species in the plasma $[1,16 \bullet]$. Chylomicrons deliver triglycerides absorbed from the diet to adipose tissue, skeletal muscle, and cardiac muscle where the enzyme lipoprotein lipase (LPL) breaks down the triglycerides (Fig. 1) $[15,35]$. In more than $80 \%$ of FCS cases, the disease is caused by an autosomal recessive variant in the $L P L$ gene $[1,36]$. The remaining FCS cases are due to autosomal recessive or compound heterozygous variants in APOA 5, APOC2, GPIHBP1, or $L M F 1$ that all affect LPL function and maturation and thereby interfere with chylomicron levels [8, 15]. More specifically, APOA5 and APOC2 are activators of LPL, LMF1 is needed for the maturation and transport of active LPL, and GPIHBP1 anchors LPL on the surface of capillary endothelial cells

The prevalence of FCS is in the range of 1:100,000 to $1: 1,000,000[1,15,37]$. The patients typically have severe hypertriglyceridemia (> $10 \mathrm{mmol} / \mathrm{L}$ ) and high fasting chylomicron levels from a young age $[16 \bullet, 36]$. As chylomicrons are large molecules, they can block pancreatic capillaries, which together with severe hypertriglyceridemia increase the risk of pancreatitis [1]. Thus, the patients often have a history of acute or recurrent pancreatitis. Other common comorbidities include lipemia retinalis, eruptive tuberous or palmar crease xanthomas, enlarged liver and spleen, and focal neurological symptoms $[8,16 \bullet]$.

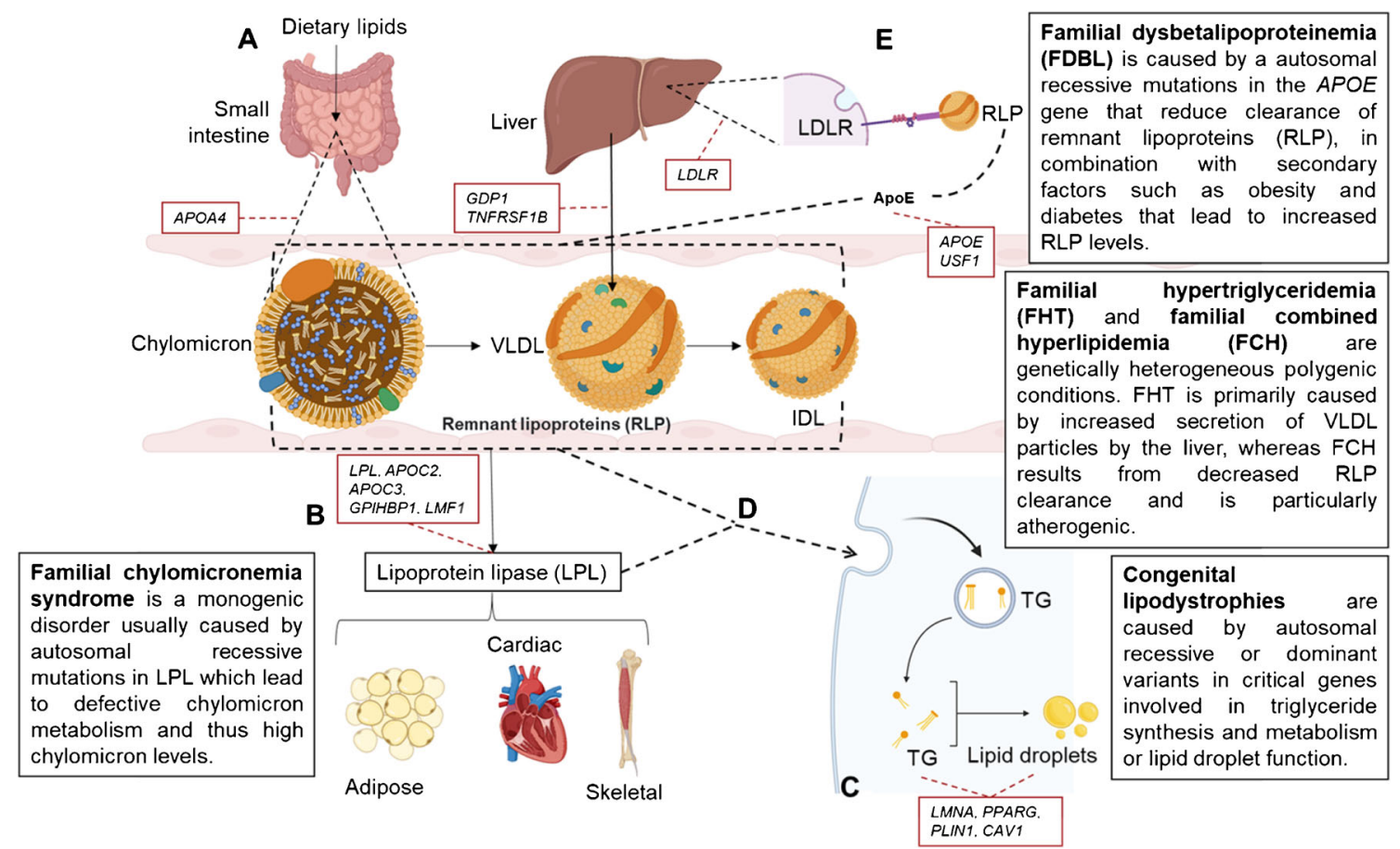

Fig. 1 Genetic mechanisms implicated in hypertriglyceridemia. A Dietary triglycerides are absorbed from the small intestine and enter the circulation packed in chylomicrons. The liver synthesizes triglycerides de novo from free fatty acids and packs them into very low-density lipoproteins (VLDLs). B The chylomicrons and VLDL deliver the triglycerides to adipose tissue, skeletal muscle, and cardiac muscle where lipoprotein lipase (LPL) cleaves triglycerides. C In the adipose tissue, triglycerides are stored within adipocyte lipid droplets. D The breakdown of triglycerides by LPL turns chylomicrons and VLDL gradually into remnant lipoproteins (RPLs). E Remnant lipoproteins are cleared by the liver via ApoE that facilitates binding of RLP to the LDL receptor (LDLR) on the liver. When RPL levels are high, RLP clearance by the liver reaches saturation and RLP molecules accumulate in plasma. Figure icons were created with BioRender.com 


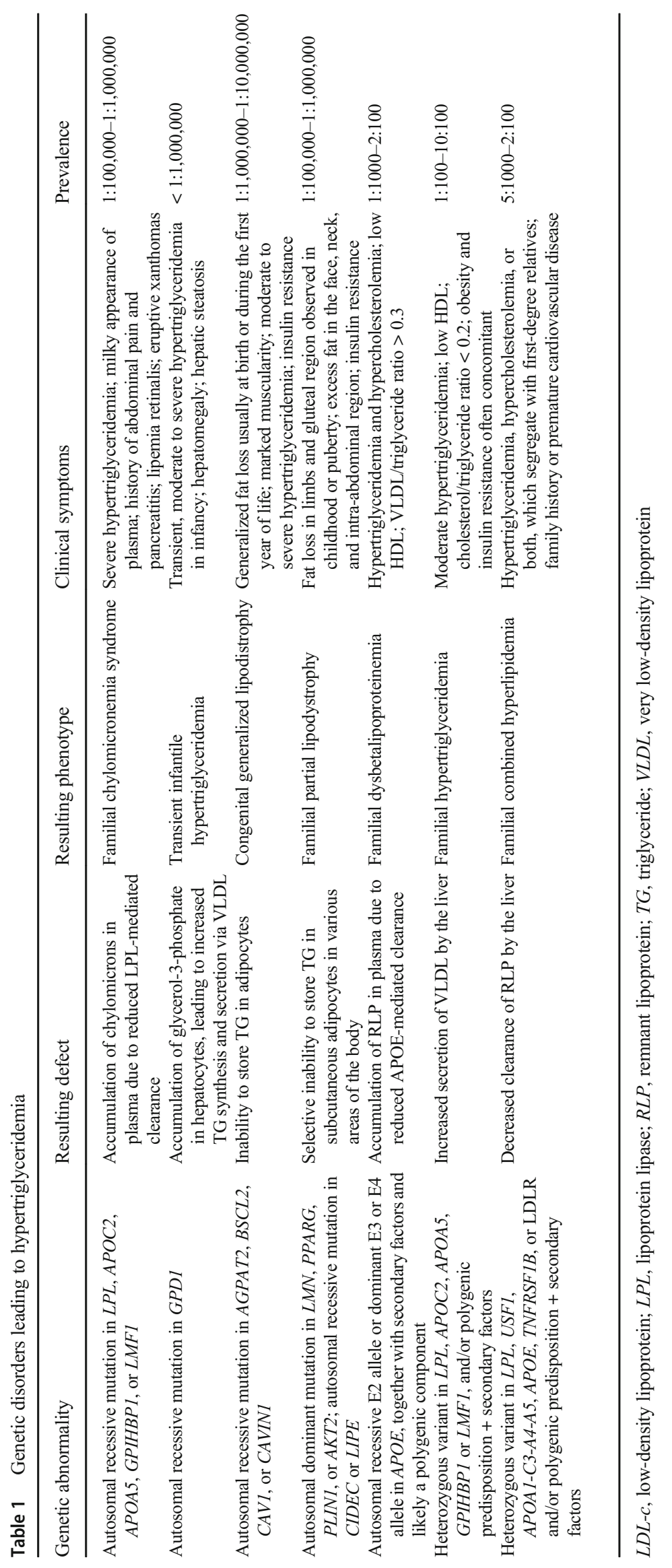


Fig. 2 The genetic architecture of hypertriglyceridemia. In very rare, monogenic disorders, a single recessive variant may cause a severe hypertriglyceridemia (red box). Rare heterozygous variants with large effects may contribute to the clustering of certain hypertriglyceridemia phenotypes in families (yellow box). In most cases of hypertriglyceridemia, the genetic basis is highly polygenic, driven by the cumulative effect of multiple common and rare variants with modest effect sizes (green box)

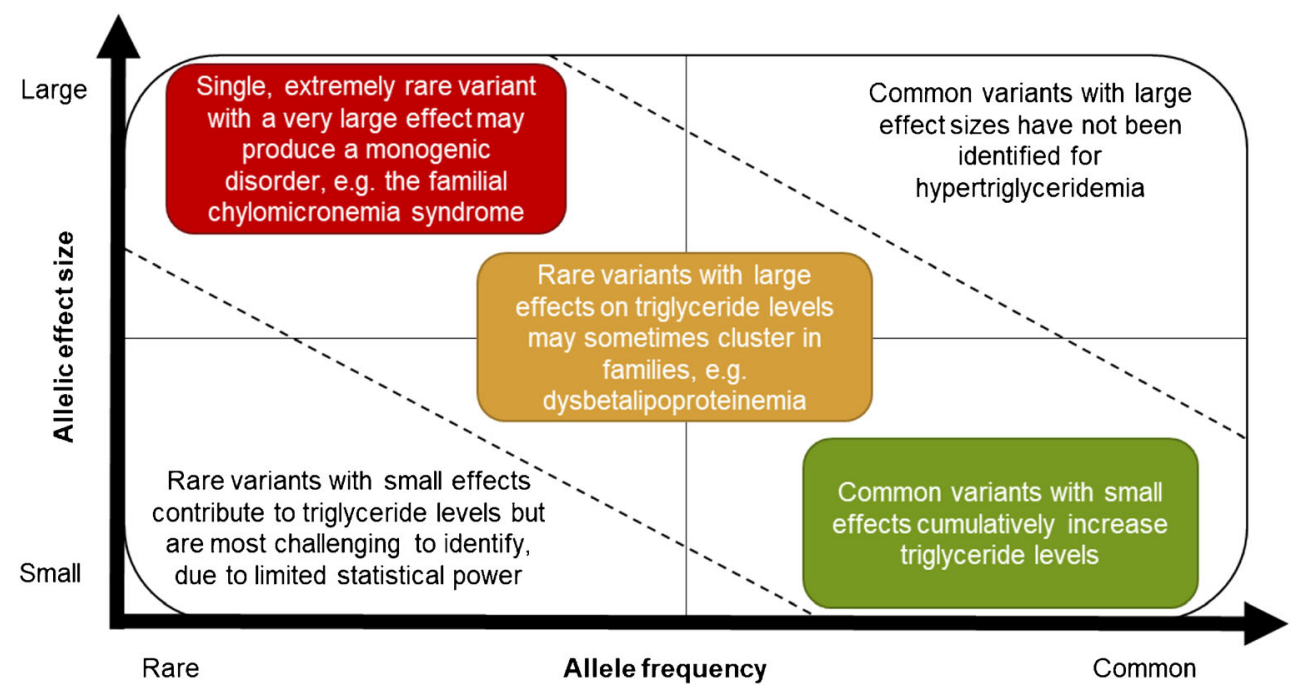

Familial Dysbetalipoproteinemia

\section{Transient Infantile Hypertriglyceridemia}

Transient infantile hypertriglyceridemia is caused by an autosomal recessive variant in the glycerol-3-phosphate dehydrogenase 1 (GPDI) gene that causes defective lipid synthesis and a high rate of triglyceride secretion by the liver. Moderate to severe hypertriglyceridemia is present at birth, but triglyceride levels normalize during growth in childhood and adolescence [38], compatible with the fact that the rate of hepatocyte triglyceride secretion is higher during the neonatal period than in adults.

\section{Congenital Lipodystrophy}

Severe hypertriglyceridemia is also commonly observed in patients with monogenic lipodystrophy syndromes, characterized by a complete or partial loss of body fat due to an inability to store fat in the adipose tissue [39, 40]. The most common types are congenital generalized lipodystrophy and familial partial lipodystrophy, caused by autosomal recessive or dominant variants in critical genes involved in lipid droplet and adipocyte function [40-42] (Table 1, Fig. 1). In lipodystrophy, triglycerides are increasingly deposited in non-adipose organs where they induce lipotoxicity. The patients show insulin resistance, diabetes, and fatty liver disease [41, 42]. Monogenic lipodystrophies are rare: the prevalence estimates range from $1: 20,000[43,44]$ to less than $1: 10,000,000[40,41]$. Lipodystrophies are not always due to direct genetic reasons - they may be acquired as a result of certain medications and autoimmune reactions [45]. While it is possible that individuals may be genetically predisposed to develop certain types of acquired lipodystrophy, this remains unproven.
Familial dysbetalipoproteinemia (FDBL) is due to autosomal homozygous recessive E2 allele in the apolipoprotein E (ApoE) gene, or in $\sim 10 \%$ of FDBL cases, a dominant E3 or E4 allele [46-48]. The underlying genetic variants are not deterministic - only 5-10\% of individuals homozygous for the ApoE2 variant develop FDBL [46, 49, 50]. The presence of secondary factors, such as diabetes or high alcohol intake, is generally required for the disease to manifest. Furthermore, there may be a polygenic component to FDBL, consisting of the cumulative effect of multiple genetic risk variants common in the population [51].

APOE functions as a ligand for remnant lipoproteins (RLP), i.e., the remnants of chylomicrons and VLDL, that bind to LDL receptors and related proteins in the liver - a process that removes RLP from the circulation [52, 53] (Fig. 1). Thus, as a result of defective APOE, the patients have reduced clearance of RLP. As high RLP levels are strongly atherogenic, FDBL can lead to premature cardiovascular disease $[46,47,54]$. Patients with FDBL show both elevated triglyceride and total cholesterol levels, generally in the range of 7 to $10 \mathrm{mmol} / \mathrm{L}$, as well as decreased HDL concentration and a VLDL/triglyceride ratio greater than $0.3[49,55,56]$. Palmar xanthomas are common $[1,56]$. The prevalence of FDBL may be up to $1-2 \%$ in the general population, but the condition is often undiagnosed [54, 57].

\section{Familial Hypertriglyceridemia and Combined Hyperlipidemia}

Familial hypertriglyceridemia (FHT) and familial combined hyperlipidemia $(\mathrm{FCH})$ are common conditions that sometimes 
cluster in families. Early studies of extended families inferred that FHT and FCH may show autosomal dominant inheritance, attributing these conditions to monogenic mutations [58]. However, subsequent investigations revealed that FHT and FCH are of polygenic origin, resulting from the cumulative effect of multiple common variants with small effects on triglyceride levels [34, 51, 59] and/or rare (minor allele frequency $<1 \%$ ) heterozygous variants with larger effect sizes on triglyceride levels [59, 60] (Fig. 2). The genes for which heterozygous variants have been implicated in FHT include $L P L[61,62], A P O A 5, A P O C 2$, GPIHBP1, and LMF1 [63] which influence LPL maturation and function. The genes implicated in $\mathrm{FCH}$ include LPL [64-66], the gene-cluster APOA1-C3-A4-A5 [67-70], APOE [71], USF1 [72], TNFRSF1B [73], and $L D L R$ [74] which affect LPL maturation and function, clearance of RLP, or VLDL secretion from the liver. Both FHT and FCH are usually accompanied by secondary factors that exacerbate the elevated triglyceride levels, such as diabetes or high alcohol intake [75]. While we refer to FHT and FCH as "familial" conditions in order to be consistent with previous literature, we note that this term is misleading as it implies a monogenic disorder [76].

In FHT, triglyceride levels are usually moderately elevated and LDL cholesterol and ApoB levels are normal or low, HDL cholesterol levels are decreased, and cholesterol:triglyceride ratio is below 1:5 [12]. It has been estimated that FHT may be present in up to 5$10 \%$ of the adult population. FCH is less common, with an estimated prevalence of $0.5-2 \%$ of the adult population [77], and more variable in terms of phenotypic expression. It involves hypertriglyceridemia, hypercholesterolemia, or both that segregate with first-degree relatives $[11,78,79 \cdot \bullet, 80]$. It is important to distinguish between FHT and FCH, because the latter is generally more atherogenic and associated with a severe increase in cardiovascular risk. Thus, the treatment also needs to be more aggressive [81].

FHT and FCH differ in etiology. FHT is primarily caused by increased secretion of VLDL particles by the liver, whereas FCH results primarily from the accumulation of RLP molecules due to decreased RLP clearance (Fig. 1). Increased RLP levels are strongly atherogenic: RLP may enter the arterial wall and become engulfed by lipid-laden macrophages, also called foam cells. If foam cells erupt, the cholesterol embodied by RLP is released and may accumulate in the arteries. Furthermore, high levels of plasma RLP result in the release of low-grade inflammation molecules, accumulation of free radicals, injuries of the endothelium, and increased endothelium permeability, causing edema and necrosis $[52,82]$.
Polygenic Hypertriglyceridemia in the GWAS Era

In the general population, genetic predisposition to moderate hypertriglyceridemia usually reflects the cumulative effect of multiple independent genetic variants on triglyceride levels [79, 83-85]. An increase in triglyceride-increasing allele load is associated with higher levels of triglycerides, and this genetic predisposition is exacerbated by secondary factors such as diet, alcohol, certain medications $[1,16 \bullet, 86]$, and concomitant metabolic conditions such as obesity, fatty liver disease, and diabetes [87]. In twin and family studies, the estimated heritability of triglyceride levels - the proportion of variability explained by genetic variation - has ranged between 40 and $60 \%$ [88], while the remaining variability is attributed to non-genetic factors.

An approach that has been found very successful for identifying genetic loci associated with elevated triglyceride levels in the general population is to perform a genome-wide association study (GWAS). In GWAS, genetic variants across the genome are screened for their association with triglyceride levels in a standardized manner, and variants reaching genome-wide significant $\mathrm{P}$ values $\left(\mathrm{P}<5 \times 10^{-8}\right.$, corresponding to Bonferroni correction for $1,000,000$ tests) are claimed as robustly associated with triglyceride levels. Thus far, $>10$ GWAS or gene exome-wide screens of triglyceride levels have been performed, which have included up to 600,000 individuals and identified more than 300 genetic loci associated with triglyceride levels with genome-wide significance $[17-22,23 \cdot \bullet, 24-33,89]$. The majority of these loci have been identified in individuals of European genetic ancestry. A handful of loci have been identified in individuals of African and Asian or Hispanic genetic ancestry [23••, 24-27, 90].

The vast majority ( $>90 \%$ ) of the variants identified thus far are common in the population (minor allele frequency $>1 \%$ ) and have effect sizes ranging from $0.01(\sim 2 \mathrm{mg} / \mathrm{dL})$ to 0.26 $(\sim 42 \mathrm{mg} / \mathrm{dL})$ standard deviation increase in plasma triglyceride levels per allele. The GWASs have also identified some rare variants with effect sizes up to $1.27(\sim 205 \mathrm{mg} / \mathrm{dL})$ standard deviation increase in triglyceride levels per allele. Identification of rare genetic variants is expected to be improved through expanded sample sizes and the application of whole-exome and whole-genome sequencing methods that are able to capture rare variants not covered by commercial genotyping arrays [89].

The GWASs performed thus far have implicated several genes already known to be involved in monogenic and familial hypertriglyceridemias, identifying associations of common variants proximal to $L P L, A P O E, A P O C 2$, and the APOA1C3-A4-A5 cluster with triglyceride levels [23••]. Pathway enrichment analyses applied on the GWAS summary results have demonstrated that polygenic hypertriglyceridemia is enriched with known mechanisms related to triglyceride 
metabolism, such as the regulation of LPL, acylglycerol homeostasis, cholesterol transport and storage, and triglyceriderich lipoprotein metabolism, transportation, and catabolism. However, the GWASs have not only identified already known genes and mechanisms but have also highlighted multiple novel genes that have not been previously known to be involved in the regulation of blood triglyceride levels. This has provided new insights into the biology underlying hypertriglyceridemia and opened avenues for the development of more effective triglyceride-lowering drugs by targeting the implicated genes [91]. The vast majority of the variants identified in GWAS are located in intronic or intergenic regions and the causal genes remain mostly unconfirmed. Pinpointing the causal gene or genes in each associated genetic region will require extensive follow up in experimental studies in vitro and/or in vivo to validate the link between the associated genetic variant or region and modified function of the candidate gene.

To study the combined influence of the triglycerideincreasing genetic loci identified in GWAS on hypertriglyceridemias, genetic variants have been combined into polygenic scores [92] which have been reported to explain up to $19.6 \%$ of the variability in triglyceride levels in the general population [93]. Studies applying polygenic scores on FHT and FCH have revealed that the scores are highly enriched in the affected patients, indicating a polygenic basis for these conditions $[78,85]$. Polygenic scores have also been applied to try predict hypertriglyceridemia in the general population; however thus far without substantial success. It has become clear that the scores will need to be used in combination with non-genetic factors to increase predictability [94].

In recent years, the genetic loci identified in GWAS have been utilized in Mendelian randomization studies to assess the causal influences of high triglyceride levels on disease traits. Mendelian randomization studies build on the random allocation of genotypes at conception, which makes it possible to assign individuals according to higher or lower genetically determined triglyceride levels in a randomized manner, equivalent to a randomized clinical trial. This reduces the influence of confounding and reverse causality on observational results. Mendelian randomization studies have indicated that higher triglyceride levels are causally associated with higher risk of coronary heart disease and hypertension in the general population $[95,96 \bullet$.

\section{Future Perspectives}

Hypertriglyceridemia is usually asymptomatic and therefore often undiagnosed and untreated. This elevates the risk for early cardiovascular disease onset and pancreatitis. Being able to early identify the individuals who are at high risk to develop severe hypertriglyceridemia would be valuable, as these individuals could then be screened further and treated to reduce the risk of cardiovascular events and pancreatitis later in life.

Despite recent advances in genetic testing, genetic tests are not yet commonly applied in to identify hypertriglyceridemias [97]. In the coming years, decreasing costs may make these tests more readily available for early genetic screening and counseling. The success of GWAS in identifying genetic loci associated with triglyceride levels have raised interest towards the use of polygenic scores for early prediction and prevention of severe hypertriglyceridemia. However, even though polygenic scores can now explain up to one-fifth of the variability in triglyceride levels in the population [93], the scores have not yet shown value for clinical practice. Furthermore, as GWASs have mostly been performed in adults of European ancestry, the polygenic scores developed thus far are not directly generalizable to individuals of other genetic ancestries. In the coming years, the predictive performance of polygenic scores may be increased through the discovery of additional genetic loci associated with triglyceride levels in diverse genetic ancestries, and by combining polygenic scores with information on non-genetic factors.

Hypertriglyceridemia can be treated pharmacologically with statins, fibrates, niacin and omega-3 acids, and nonpharmacologically with lifestyle changes, such as calorierestricted diets and exercise $[1,8]$. The goal of the treatments is to reduce the risk of cardiovascular disease and pancreatitis. The triglyceride-lowering treatments are currently applied in "a one-size-fits-all" manner to all individuals [98]. However, the pathogenic mechanisms that elevate triglyceride levels are heterogeneous between individuals. The discoveries in GWAS may provide opportunities for tailoring triglyceridelowering treatments to specific individuals or groups by identifying the specific genetic mechanisms that are affected.

Genetic findings have also opened avenues for the development of more effective triglyceride-lowering drugs, by targeting the identified genes and pathways [91]. For instance, whole-exome sequencing studies solidified the causal role of $A P O C 3$ in the regulation of triglyceride levels and risk of coronary artery disease. Antisense oligonucleotides that target APOC 3 mRNA were recently approved by the European Medicines Agency for the treatment of FCS. As another successful example, GWASs have identified both common and rare variants in the angiopoietin-like 3 (ANGPTL3) gene associated with plasma triglyceride levels. The ANGTPL3 variants inhibit LPL activity, and recent Phase 1 trials for monoclocal antibodies that similarly inhibit ANGTPL3 demonstrated that the antibodies reduce triglyceride levels in hypertriglyceridemic patients [99]. The antibodies have previously been approved by the U.S. Food and Drug Administration for the treatment of homozygous familial hypercholesterolemia. Finally, GWASs for plasma triglycerides have implicated several genes involved in hepatic lipogenesis, 
a previously underappreciated pathway in the regulation of plasma triglyceride levels [91]. However, most of the identified genes are not associated with risk of cardiovascular disease, suggesting that hepatic lipogenesis may not be a sufficient target to prevent cardiovascular events [91].

At present, the causal genes and their functions remain unknown for the vast majority of the loci identified in GWAS, which hinders translational efforts. The increased use of whole-exome and whole-genome sequencing may lead to enhanced identification of rare coding variants that lead to loss or gain of gene function and show large effect sizes on triglyceride levels. Such variants have particularly high potential for drug targeting [89].

\section{Conclusions}

Hypertriglyceridemia can have a monogenic or polygenic basis. In monogenic hypertriglyceridemias, which are extremely rare, homozygous genetic variants leading to dysfunctional proteins involved in triglyceride metabolism lead to severe hypertriglyceridemia. In the vast majority of cases, however, hypertriglyceridemia is of complex polygenic origin, whereby the cumulative effects of multiple genetic variants, together with non-genetic factors, contribute to elevated levels of triglycerides. Over recent years, familial hypertriglyceridemia and combined hyperlipidemia, previously considered monogenic, have been found to be mainly polygenic conditions.

In GWAS performed to date, $>300$ independent genetic loci have been found associated with plasma triglyceride levels, revealing new biology and opening avenues for the development of more effective treatments against hypertriglyceridemia. Successful examples of GWAS-identified targets for triglyceride-lowering drugs include $A P O C 3$ and $A N G P T L 3$. However, for the vast majority of identified loci, the causal genes remain unknown, warranting extensive efforts to experimentally validate the causal genes and their functions in vitro and in vivo.

The GWAS findings may allow the development of early screening tools for severe hypertriglyceridemia. At present, nearly all loci currently pertain to European ancestry, and more efforts are required to increase knowledge on the genetic basis of hypertriglyceridemia in non-European ancestries. Another emerging possibility is to use genetic information to better tailor triglyceride-lowering treatments for individual patients, based on the specific genetic mechanisms that are affected.

Funding The Novo Nordisk Foundation Center for Basic Metabolic Research is an independent research center at the University of Copenhagen partially funded by an unrestricted donation from the Novo Nordisk Foundation (NNF18CC0034900). Germán D. Carrasquilla and Malene Revsbech Christiansen were supported by grants from the Danish Diabetes Academy which is funded by the Novo Nordisk Foundation (NNF17SA0031406). Germán D. Carrasquilla received funding from the European Union's Horizon 2020 Research and Innovation Programme under the Marie Sklodowska-Curie grant agreement No 846502 .

\section{Declarations}

Conflict of Interest The authors declare that they have no conflict of interest.

Human and Animal Rights and Informed Consent This article does not contain any studies with human or animal subjects performed by any of the authors.

Open Access This article is licensed under a Creative Commons Attribution 4.0 International License, which permits use, sharing, adaptation, distribution and reproduction in any medium or format, as long as you give appropriate credit to the original author(s) and the source, provide a link to the Creative Commons licence, and indicate if changes were made. The images or other third party material in this article are included in the article's Creative Commons licence, unless indicated otherwise in a credit line to the material. If material is not included in the article's Creative Commons licence and your intended use is not permitted by statutory regulation or exceeds the permitted use, you will need to obtain permission directly from the copyright holder. To view a copy of this licence, visit http://creativecommons.org/licenses/by/4.0/.

\section{References}

Papers of particular interest, published recently, have been highlighted as:

- Of importance

•- Of major importance

1. Simha V. Management of hypertriglyceridemia. BMJ. 2020;371: m3109.

2. Laufs U, Parhofer KG, Ginsberg HN, Hegele RA. Clinical review on triglycerides. Eur Heart J. 2020;41(1):99-109c.

3. Nordestgaard BG, Varbo A. Triglycerides and cardiovascular disease. Lancet. 2014;384(9943):626-35.

4. Arca M, Veronesi C, D'Erasmo L, et al. Association of hypertriglyceridemia with all-cause mortality and atherosclerotic cardiovascular events in a low-risk Italian population: the TG-REAL retrospective cohort analysis. J Am Heart Assoc. 2020;9(19):e015801.

5. Emerging Risk Factors C, Di Angelantonio E, Sarwar N, et al. Major lipids, apolipoproteins, and risk of vascular disease. JAMA. 2009;302(18):1993-2000.

6. Sarwar N, Danesh J, Eiriksdottir G, Sigurdsson G, Wareham N, Bingham S, et al. Triglycerides and the risk of coronary heart disease: 10,158 incident cases among 262,525 participants in 29 Western prospective studies. Circulation. 2007;115(4):450-8.

7. Pedersen SB, Langsted A, Nordestgaard BG. Nonfasting mild-tomoderate hypertriglyceridemia and risk of acute pancreatitis. JAMA Intern Med. 2016;176(12):1834-42.

8. Goldberg RB, Chait A. A comprehensive update on the chylomicronemia syndrome. Front Endocrinol (Lausanne). 2020;11:593931.

9. Case BC, Bress AP, Kolm P, Philip S, Herrick JS, Granowitz CB, et al. The economic burden of hypertriglyceridemia among US 
adults with diabetes or atherosclerotic cardiovascular disease on statin therapy. J Clin Lipidol. 2019;13(5):754-61.

10. Miller M, Stone NJ, Ballantyne C, Bittner V, Criqui MH, Ginsberg $\mathrm{HN}$, et al. Triglycerides and cardiovascular disease: a scientific statement from the American Heart Association. Circulation. 2011;123(20):2292-333.

11. Mach F, Baigent C, Catapano AL, Koskinas KC, Casula M, Badimon L, et al. 2019 ESC/EAS guidelines for the management of dyslipidaemias: lipid modification to reduce cardiovascular risk. Eur Heart J. 2020;41(1):111-88.

12. Authors/Task Force M, Catapano AL, Graham I, et al. ESC/EAS guidelines for the management of dyslipidaemias: the Task Force for the management of dyslipidaemias of the European Society of Cardiology (ESC) and European Atherosclerosis Society (EAS) developed with the special contribution of the European Assocciation for Cardiovascular Prevention \& Rehabilitation (EACPR). Atherosclerosis. 2016;2016(253):281-344.

13. Berglund L, Brunzell JD, Goldberg AC, Goldberg IJ, Sacks F, Murad MH, et al. Evaluation and treatment of hypertriglyceridemia: an Endocrine Society clinical practice guideline. J Clin Endocrinol Metab. 2012;97(9):2969-89.

14. Chyzhyk V, Kozmic S, Brown AS, Hudgins LC, Starc TJ, Davila $\mathrm{AD}$, et al. Extreme hypertriglyceridemia: genetic diversity, pancreatitis, pregnancy, and prevalence. J Clin Lipidol. 2019;13(1):89-99.

15. Ramasamy I. Update on the molecular biology of dyslipidemias. Clin Chim Acta. 2016;454:143-85.

16. Dron JS, Hegele RA. Genetics of hypertriglyceridemia. Front Endocrinol (Lausanne). 2020;11:455 Targeted sequencing of DNA from patients with severe hypertriglyceridemia shows that $1 / 3$ of the patients have a high polygenic score for hypertriglyceridemia, $\sim 15 \%$ carry rare heterozygous variants, and $\sim 1 \%$ have monogenic hypertriglyceridemia.

17. Willer CJ, Schmidt EM, Sengupta S, Peloso GM, Gustafsson S, Kanoni S, et al. Discovery and refinement of loci associated with lipid levels. Nat Genet. 2013;45(11):1274-83.

18. Below JE, Parra EJ, Gamazon ER, Torres J, Krithika S, Candille S, et al. Meta-analysis of lipid-traits in Hispanics identifies novel loci, population-specific effects, and tissue-specific enrichment of eQTLs. Sci Rep. 2016;6:19429.

19. Davis JP, Huyghe JR, Locke AE, Jackson AU, Sim X, Stringham $\mathrm{HM}$, et al. Common, low-frequency, and rare genetic variants associated with lipoprotein subclasses and triglyceride measures in Finnish men from the METSIM study. PLoS Genet. 2017;13(10): e1007079.

20. Liu DJ, Peloso GM, Yu H, et al. Exome-wide association study of plasma lipids in $>300,000$ individuals. Nat Genet. 2017;49(12): $1758-66$.

21. Nagy R, Boutin TS, Marten J, Huffman JE, Kerr SM, Campbell A, et al. Exploration of haplotype research consortium imputation for genome-wide association studies in 20,032 Generation Scotland participants. Genome Med. 2017;9(1):23.

22. Southam L, Gilly A, Suveges D, et al. Whole genome sequencing and imputation in isolated populations identify genetic associations with medically-relevant complex traits. Nat Commun. 2017;8: 15606.

23.• Klarin D, Damrauer SM, Cho K, et al. Genetics of blood lipids among 300,000 multi-ethnic participants of the Million Veteran Program. Nat Genet. 2018;50(11):1514-23 The largest genomewide association study of triglyceride levels published to date, including up to 598,000 adults.

24. Kanai M, Akiyama M, Takahashi A, Matoba N, Momozawa Y, Ikeda $\mathrm{M}$, et al. Genetic analysis of quantitative traits in the Japanese population links cell types to complex human diseases. Nat Genet. 2018;50(3):390-400.

25. Lu X, Huang J, Mo Z, He J, Wang L, Yang X, et al. Genetic susceptibility to lipid levels and lipid change over time and risk of incident hyperlipidemia in Chinese populations. Circ Cardiovasc Genet. 2016;9(1):37-44.

26. Lu X, Peloso GM, Liu DJ, et al. Exome chip meta-analysis identifies novel loci and East Asian-specific coding variants that contribute to lipid levels and coronary artery disease. Nat Genet. 2017;49(12):1722-30.

27. Spracklen CN, Chen P, Kim YJ, Wang X, Cai H, Li S, et al. Association analyses of East Asian individuals and trans-ancestry analyses with European individuals reveal new loci associated with cholesterol and triglyceride levels. Hum Mol Genet. 2017;26(9): $1770-84$.

28. van Leeuwen EM, Sabo A, Bis JC, Huffman JE, Manichaikul A, Smith AV, et al. Meta-analysis of 49549 individuals imputed with the 1000 Genomes Project reveals an exonic damaging variant in ANGPTL4 determining fasting TG levels. J Med Genet. 2016;53(7):441-9.

29. Kilpelainen TO, Bentley AR, Noordam R, et al. Multi-ancestry study of blood lipid levels identifies four loci interacting with physical activity. Nat Commun. 2019;10(1):376.

30. de Vries PS, Brown MR, Bentley AR, Sung YJ, Winkler TW, Ntalla I, et al. Multiancestry genome-wide association study of lipid levels incorporating gene-alcohol interactions. Am J Epidemiol. 2019;188(6):1033-54.

31. Bentley AR, Sung YJ, Brown MR, et al. Multi-ancestry genomewide gene-smoking interaction study of 387,272 individuals identifies new loci associated with serum lipids. Nat Genet. 2019;51(4): 636-48.

32. Noordam R, Bos MM, Wang H, Winkler TW, Bentley AR, Kilpeläinen TO, et al. Multi-ancestry sleep-by-SNP interaction analysis in 126,926 individuals reveals lipid loci stratified by sleep duration. Nat Commun. 2019;10(1):5121.

33. $\mathrm{Hu} \mathrm{Y,} \mathrm{Graff} \mathrm{M,} \mathrm{Haessler} \mathrm{J,} \mathrm{Buyske} \mathrm{S,} \mathrm{Bien} \mathrm{SA,} \mathrm{Tao} \mathrm{R,} \mathrm{et} \mathrm{al.}$ Minority-centric meta-analyses of blood lipid levels identify novel loci in the Population Architecture using Genomics and Epidemiology (PAGE) study. PLoS Genet. 2020;16(3):e1008684.

34. Nabirotchkin S, Peluffo AE, Rinaudo P, Yu J, Hajj R, Cohen D. Next-generation drug repurposing using human genetics and network biology. Curr Opin Pharmacol. 2020;51:78-92.

35. Kane JP, Hardman DA, Paulus HE. Heterogeneity of apolipoprotein B: isolation of a new species from human chylomicrons. Proc Natl Acad Sci U S A. 1980;77(5):2465-9.

36. Dron JS, Hegele RA. Genetics of triglycerides and the risk of atherosclerosis. Curr Atheroscler Rep. 2017;19(7):31.

37. Patni N, Li X, Adams-Huet B, Garg A. The prevalence and etiology of extreme hypertriglyceridemia in children: data from a tertiary children's hospital. J Clin Lipidol. 2018;12(2):305-10.

38. Basel-Vanagaite L, Zevit N, Har Zahav A, et al. Transient infantile hypertriglyceridemia, fatty liver, and hepatic fibrosis caused by mutated GPD1, encoding glycerol-3-phosphate dehydrogenase 1. Am J Hum Genet. 2012;90(1):49-60.

39. Araujo-Vilar D, Santini F. Diagnosis and treatment of lipodystrophy: a step-by-step approach. J Endocrinol Investig. 2019;42(1):61-73.

40. Knebel B, Muller-Wieland D, Kotzka J. Lipodystrophies-disorders of the fatty tissue. Int J Mol Sci. 2020;21(22):8778.

41. Lightbourne M, Brown RJ. Genetics of lipodystrophy. Endocrinol Metab Clin N Am. 2017;46(2):539-54.

42. Hussain I, Garg A. Lipodystrophy syndromes. Endocrinol Metab Clin N Am. 2016;45(4):783-97.

43. Garg A. Acquired and inherited lipodystrophies. N Engl J Med. 2004;350(12):1220-34.

44. Gonzaga-Jauregui C, Ge W, Staples J, van Hout C, Yadav A, Colonie R, et al. Clinical and molecular prevalence of lipodystrophy in an unascertained large clinical care cohort. Diabetes. 2020;69(2):249-58. 
45. Chan JL, Oral EA. Clinical classification and treatment of congenital and acquired lipodystrophy. Endocr Pract. 2010;16(2):310-23.

46. Utermann G, Hees M, Steinmetz A. Polymorphism of apolipoprotein $\mathrm{E}$ and occurrence of dysbetalipoproteinaemia in man. Nature. 1977;269(5629):604-7.

47. Havel RJ, Chao Y, Windler EE, Kotite L, Guo LS. Isoprotein specificity in the hepatic uptake of apolipoprotein $\mathrm{E}$ and the pathogenesis of familial dysbetalipoproteinemia. Proc Natl Acad Sci U S A. 1980;77(7):4349-53.

48. Zannis VI, Just PW, Breslow JL. Human apolipoprotein E isoprotein subclasses are genetically determined. Am J Hum Genet. 1981;33(1):11-24.

49. Schaefer JR. Unraveling hyperlipidemia type III (dysbetalipoproteinemia), slowly. Eur J Hum Genet. 2009;17(5): $541-2$.

50. Utermann G, Vogelberg KH, Steinmetz A, et al. Polymorphism of apolipoprotein E. II. Genetics of hyperlipoproteinemia type III. Clin Genet. 1979;15(1):37-62.

51. Johansen CT, Wang J, Lanktree MB, McIntyre AD, Ban MR, Martins RA, et al. An increased burden of common and rare lipid-associated risk alleles contributes to the phenotypic spectrum of hypertriglyceridemia. Arterioscler Thromb Vasc Biol. 2011;31(8):1916-26.

52. Reiner Z. Hypertriglyceridaemia and risk of coronary artery disease. Nat Rev Cardiol. 2017;14(7):401-11.

53. Mahley RW, Hui DY, Innerarity TL, Weisgraber KH. Two independent lipoprotein receptors on hepatic membranes of dog, swine, and man. Apo-B,E and apo-E receptors. J Clin Invest. 1981;68(5): 1197-206.

54. Koopal C, Marais AD, Visseren FL. Familial dysbetalipoproteinemia: an underdiagnosed lipid disorder. Curr Opin Endocrinol Diabetes Obes. 2017;24(2):133-9.

55. Pallazola VA, Sathiyakumar V, Park J, Vakil RM, Toth P, LazoElizondo M, et al. Modern prevalence of dysbetalipoproteinemia (Fredrickson-Levy-Lees type III hyperlipoproteinemia). Arch Med Sci. 2020;16(5):993-1003.

56. Morganroth J, Levy RI, Fredrickson DS. The biochemical, clinical, and genetic features of type III hyperlipoproteinemia. Ann Intern Med. 1975;82(2):158-74.

57. Sathiyakumar V, Pallazola VA, Park J, Vakil RM, Toth P, LazoElizondo M, et al. Modern prevalence of the Fredrickson-LevyLees dyslipidemias: findings from the Very Large Database of Lipids and National Health and Nutrition Examination Survey. Arch Med Sci. 2020;16(6):1279-87.

58. Goldstein JL, Schrott HG, Hazzard WR, Bierman EL, Motulsky AG. Hyperlipidemia in coronary heart disease. II. Genetic analysis of lipid levels in 176 families and delineation of a new inherited disorder, combined hyperlipidemia. J Clin Invest. 1973;52(7): $1544-68$.

59. Johansen CT, Wang J, McIntyre AD, et al. Excess of rare variants in non-genome-wide association study candidate genes in patients with hypertriglyceridemia. Circ Cardiovasc Genet. 2012;5(1):6672.

60. Hegele RA, Ban MR, Hsueh N, Kennedy BA, Cao H, Zou GY, et al. A polygenic basis for four classical Fredrickson hyperlipoproteinemia phenotypes that are characterized by hypertriglyceridemia. Hum Mol Genet. 2009;18(21):4189-94.

61. Pingitore P, Lepore SM, Pirazzi C, Mancina RM, Motta BM, Valenti L, et al. Identification and characterization of two novel mutations in the LPL gene causing type I hyperlipoproteinemia. J Clin Lipidol. 2016;10(4):816-23.

62. Kersten S. Physiological regulation of lipoprotein lipase. Biochim Biophys Acta. 2014;1841(7):919-33.

63. Bello-Chavolla OY, Kuri-Garcia A, Rios-Rios M, et al. Familial combined hyperlipidemia: current knowledge, perspectives, and controversies. Rev Investig Clin. 2018;70(5):224-36.
64. Yang WS, Nevin DN, Iwasaki L, Peng R, Brown BG, Brunzell JD, et al. Regulatory mutations in the human lipoprotein lipase gene in patients with familial combined hyperlipidemia and coronary artery disease. J Lipid Res. 1996;37(12):2627-37.

65. Campagna F, Montali A, Baroni MG, Maria AT, Ricci G, Antonini $\mathrm{R}$, et al. Common variants in the lipoprotein lipase gene, but not those in the insulin receptor substrate-1, the beta3-adrenergic receptor, and the intestinal fatty acid binding protein- 2 genes, influence the lipid phenotypic expression in familial combined hyperlipidemia. Metabolism. 2002;51(10):1298-305.

66. Reymer PW, Groenemeyer BE, Gagne E, et al. A frequently occurring mutation in the lipoprotein lipase gene (Asn291Ser) contributes to the expression of familial combined hyperlipidemia. Hum Mol Genet. 1995;4(9):1543-9.

67. Groenendijk M, Cantor RM, Funke H, Dallinga-Thie GM. Two newly identified SNPs in the APO AI-CIII intergenic region are strongly associated with familial combined hyperlipidaemia. Eur J Clin Investig. 2001;31(10):852-9.

68. Liu ZK, Hu M, Baum L, Thomas GN, Tomlinson B. Associations of polymorphisms in the apolipoprotein $\mathrm{A} 1 / \mathrm{C} 3 / \mathrm{A} 4 / \mathrm{A} 5$ gene cluster with familial combined hyperlipidaemia in Hong Kong Chinese. Atherosclerosis. 2010;208(2):427-32.

69. Xu CF, Talmud P, Schuster H, Houlston R, Miller G, Humphries S. Association between genetic variation at the APO AI-CIII-AIV gene cluster and familial combined hyperlipidaemia. Clin Genet. 1994;46(6):385-97.

70. van der Vleuten GM, Isaacs A, Zeng WW, ter Avest E, Talmud PJ, Dallinga-Thie GM, et al. Haplotype analyses of the APOA5 gene in patients with familial combined hyperlipidemia. Biochim Biophys Acta. 2007;1772(1):81-8.

71. Solanas-Barca M, de Castro-Oros I, Mateo-Gallego R, et al. Apolipoprotein E gene mutations in subjects with mixed hyperlipidemia and a clinical diagnosis of familial combined hyperlipidemia. Atherosclerosis. 2012;222(2):449-55.

72. Pajukanta P, Lilja HE, Sinsheimer JS, Cantor RM, Lusis AJ, Gentile M, et al. Familial combined hyperlipidemia is associated with upstream transcription factor 1 (USF1). Nat Genet. 2004;36(4):371-6.

73. Geurts JM, Janssen RG, van Greevenbroek MM, van der Kallen C, Cantor RM, Bu X, et al. Identification of TNFRSF1B as a novel modifier gene in familial combined hyperlipidemia. Hum Mol Genet. 2000;9(14):2067-74.

74. Civeira F, Jarauta E, Cenarro A, García-Otín AL, Tejedor D, Zambón D, et al. Frequency of low-density lipoprotein receptor gene mutations in patients with a clinical diagnosis of familial combined hyperlipidemia in a clinical setting. J Am Coll Cardiol. 2008;52(19):1546-53.

75. Goyal A, Cusick AS, Bansal P: Familial hypertriglyceridemia. In: StatPearls. Treasure Island (FL); 2020.

76. Hegele RA, Ginsberg HN, Chapman MJ, Nordestgaard BG, Kuivenhoven JA, Averna M, et al. The polygenic nature of hypertriglyceridaemia: implications for definition, diagnosis, and management. Lancet Diabetes Endocrinol. 2014;2(8):655-66.

77. Daniels SR, Couch SC. Chapter 25 - Lipid Disorders in Children and Adolescent. In: Sperling MA Sperling (editor). Pediatric Endocrinology (Fifth Edition), Philadelphia, USA: Elsevier; 2020. pp 1004-1021

78. Ripatti P, Ramo JT, Soderlund S, et al. The contribution of GWAS loci in familial dyslipidemias. PLoS Genet. 2016;12(5):e1006078.

79. Gill PK, Dron JS, Berberich AJ, et al. Combined hyperlipidemia is genetically similar to isolated hypertriglyceridemia. J Clin Lipidol. 2021;15(1):79-87 Targeted sequencing of DNA from patients with combined hyperlipidemia and isolated hypertriglyceridemia finds that both conditions show similar enrichment of common triglyceride-increasing genetic variants. 
80. Talmud PJ, Shah S, Whittall R, Futema M, Howard P, Cooper JA, et al. Use of low-density lipoprotein cholesterol gene score to distinguish patients with polygenic and monogenic familial hypercholesterolaemia: a case-control study. Lancet. 2013;381(9874):1293301.

81. Brunzell JD. Clinical practice. Hypertriglyceridemia. N Engl J Med. 2007;357(10):1009-17.

82. Saraswathi V, Hasty AH. The role of lipolysis in mediating the proinflammatory effects of very low density lipoproteins in mouse peritoneal macrophages. J Lipid Res. 2006;47(7):1406-15.

83. De Castro-Oros I, Cenarro A, Tejedor MT, et al. Common genetic variants contribute to primary hypertriglyceridemia without differences between familial combined hyperlipidemia and isolated hypertriglyceridemia. Circ Cardiovasc Genet. 2014;7(6):814-21.

84. De Castro-Oros I, Civeira F, Pueyo MJ, et al. Rare genetic variants with large effect on triglycerides in subjects with a clinical diagnosis of familial vs nonfamilial hypertriglyceridemia. J Clin Lipidol. 2016;10(4):790-7.

85. Dron JS, Wang J, Cao H, McIntyre AD, Iacocca MA, Menard JR, et al. Severe hypertriglyceridemia is primarily polygenic. J Clin Lipidol. 2019;13(1):80-8.

86. Musambil M, Al-Rubeaan K, Al-Qasim S, Al Naqeb D, AlSoghayer A. Primary hypertriglyceridemia: a look back on the clinical classification and genetics of the disease. Curr Diabetes Rev. 2020;16(6):521-31.

87. Williams PT. Gene-environment interactions due to quantilespecific heritability of triglyceride and VLDL concentrations. Sci Rep. 2020;10(1):4486.

88. Wong MW, Thalamuthu A, Braidy N, et al. Genetic and environmental determinants of variation in the plasma lipidome of older Australian twins. Elife. 2020;9; e58954.

89. Dewey FE, Murray MF, Overton JD, et al. Distribution and clinical impact of functional variants in 50,726 whole-exome sequences from the DiscovEHR study. Science. 2016;354(6319);aaf6814.

90. Hindy G, Dornbos P, Chaffin MD, et al: Rare coding variants in 35 genes associate with circulating lipid levels-a multi-ancestry analysis of 170,000 exomes. bioRxiv. 2020, https://doi.org/10. $1101 / 2020.12 .22 .423783$

91. Bauer RC, Khetarpal SA, Hand NJ, Rader DJ. Therapeutic targets of triglyceride metabolism as informed by human genetics. Trends Mol Med. 2016;22(4):328-40.

92. Teslovich TM, Musunuru K, Smith AV, Edmondson AC, Stylianou IM, Koseki M, et al. Biological, clinical and population relevance of 95 loci for blood lipids. Nature. 2010;466(7307):707-13.

93. So HC, Sham PC. Exploring the predictive power of polygenic scores derived from genome-wide association studies: a study of 10 complex traits. Bioinformatics. 2017;33(6):886-92.

94. Lambert SA, Abraham G, Inouye M. Towards clinical utility of polygenic risk scores. Hum Mol Genet. 2019;28(R2):R133-42.

95. Do R, Willer CJ, Schmidt EM, Sengupta S, Gao C, Peloso GM, et al. Common variants associated with plasma triglycerides and risk for coronary artery disease. Nat Genet. 2013;45(11):1345-52.

96. Allara E, Morani G, Carter P, et al. Genetic determinants of lipids and cardiovascular disease outcomes: a wide-angled Mendelian randomization investigation. Circ Genom Precis Med. 2019;12(12):e002711 Mendelian randomization suggests that higher triglyceride levels are causally associated with increased risk of coronary artery disease, aortic valve stenosis, and hypertension.

97. Nawawi HM, Chua YA, Watts GF. The brave new world of genetic testing in the management of the dyslipidaemias. Curr Opin Cardiol. 2020;35(3):226-33.

98. Kushner PA, Cobble ME. Hypertriglyceridemia: the importance of identifying patients at risk. Postgrad Med. 2016;128(8):848-58.

99. Ahmad Z, Banerjee P, Hamon S, Chan KC, Bouzelmat A, Sasiela WJ, et al. Inhibition of angiopoietin-like protein 3 with a monoclonal antibody reduces triglycerides in hypertriglyceridemia. Circulation. 2019;140(6):470-86.

Publisher's Note Springer Nature remains neutral with regard to jurisdictional claims in published maps and institutional affiliations. 